\title{
LRS Bianchi Type I Cosmological Models with Perfect Fluid and Dark Energy in Bimetric Theory of Gravitation
}

\author{
M. S. Borkar ${ }^{1}$, S. S. Charjan ${ }^{2}$, V.V. Lepse ${ }^{3}$ \\ ${ }^{I}$ (Department of Mathematics, R. T. M. Nagpur University, Nagpur, India) \\ ${ }_{2}^{2}$ (Department of Mathematics, Late K. Z. S. College, Bramhani, Nagpur, India) \\ ${ }^{3}$ (Department of Mathematics, Science College, Pauni, Bhandara, India)
}

\begin{abstract}
In this paper, LRS bianchi type I models in the light of perfect fluid, quintessence and standard Chaplygin gas, have been studied by solving the Rosen's field equations in bimetric theory of gravitation. It is pointed out that the geometry of perfect fluid model is coincide with the geometry of quintessence model for $\omega_{q} \in[-1,0], \omega_{q} \neq-1 / 3$ and the geometry of quintessence model for $\omega_{q}=-1 / 3$ is similar to geometry of standard Chaplygin gas model. All models are expanding with accelerating expansion. Other geometrical and physical properties of the model are studied.

Our opinion supports the observational data that universe is continuously expanding with accelerating expansion.
\end{abstract}

Keywords: Cosmology, Dark Energy, Dark Matter.

\section{Introduction}

The different cosmological models with dark energy and dark matter have been taken considerable interest in General relativity. More recently, dark energy has been found to be required in order to explain the apparent accelerating expansion of the universe. It now seems that universe containing $23 \%$ dark matter and $73 \%$ dark energy in the form of energy densities and the remaining $4 \%$ is baryonic matter [1-4]. The dark matter is unknown form of matter which has clustering properties of ordinary matter and has not been yet detected. The dark energy is hypothetical form of energy acting repulsively which permits all of space and tends to accelerate the expansion of the universe. This accelerated expansion of the universe was confirmed by the observations such as type I supernovae (SNeIa) [5-7], Sloan Digital Sky Survey [8], and Wilkinson Microwave Anisotropic Probe (WMAP) [9-11]. In the light of these observations, depending on the ideas of dark energy, cosmologist proposed many candidate for dark energy such as cosmological constant, quintessence [12-13], phantom [1415], quintom [16-17], tachyons field [18-19], Chaplygin gas models [20-21] dark energy models. The cosmological constant candidate suffers from two well known problems namely the fine tuning and the cosmic coincident problems [22]. The quintessence dynamical dark energy model is combination of positive energy density and the negative pressure. Tracker field (a form of quintessence dark energy model) which provide a new motivation for the quintessence scenario [23-24]. Another alternative to dynamical dark energy model is Chaplygin gas model which is considered to be unification of dark matter and dark energy. In Chaplygin model, three different models namely standard Chaplygin gas, generalized Chaplygin gas and modified Chaplygin gas models given in [25-30].

The great success of General Relativity, however not stopped alternative theories being proposed in order to explain acceleration and existence of dark matter in the universe. One of the alternative theories among them is Rosen's bimetric theory of gravitation which is free from singularities appearing in the big-bang of cosmological models and it obeys the principle of covariance and equivalence of the general relativity [31-32]. Therefore the peoples are interested to investigate the cosmological models of the universe in bimetric theory of gravitation which is based on two matrices, one is Riemannian metric described the geometry of curved space time and second is flat metric refers to the geometry of the empty universe and described the initial forces. The Rosen's field equations in bimetric theory of gravitation are

$$
N_{i}^{j}-\frac{1}{2} N \delta_{i}^{j}=-8 \pi k T_{i}^{j}
$$

where $N_{i}^{j}=\frac{1}{2} \gamma^{\alpha \beta}\left(g^{s j} g_{s i \mid \alpha}\right)_{\mid \beta}, N=g^{i j} N_{i j}$ is the Rosen scalar and let $k=1$. The vertical bar $(\mid)$ stands for $\gamma$-covariant differentiation, where $g=\operatorname{det}\left(g_{i j}\right)$ and $\gamma=\operatorname{det}\left(\gamma_{i j}\right)$. Many researchers have been developed the theory and investigated various cosmological models of the universe, in bimetric theory of gravitation and studied their behavior geometrically and physically [33-48]. A locally rotationally symmetric 
(LRS) having much more contribution in the physics of spatial homogeneous cosmological model and therefore we plan to study a LRS bianchi type I cosmological model in related to perfect fluid with quintessence and standard Chaplygin gas dark energy in this research note. The model is filled with perfect fluid $p=\omega \rho, 0 \leq \omega \leq 1$. For quintessence, we consider $p_{q}=\omega_{q} \rho_{q}$, where $\omega_{q} \in[-1,0]$ and for standard Chaplygin gas dark energy model, the equation of state is to be assumed $p_{c}=-\mu / \rho_{c}$, where $\mu$ is constant.

In view of these considerations, LRS bianchi type I models have been studied and it is realized that: i) The model with perfect fluid has volumetric exponential expansion and is always supported isotropize, shear less dust universe with accelerating expansion. ii) The quintessence dark energy model with $\omega_{q} \in[-1,0]$ and $\omega_{q} \neq-1 / 3$, having the similar physics as that of perfect fluid model in regards of geometry and all its physical parameters. The quintessence dark energy model with $\omega_{q}=-1 / 3$ represents dark energy star and it is continuously expanding and expansion has accelerating phase. iii) The Chaplygin gas model is behaves like that of dark energy model for $\omega_{q}=-1 / 3$ and having the same geometrical and physical significance as that of it.

\section{Metric And Solutions Of Field Equations}

We consider LRS bianchi type-I metric in the form

$$
d s^{2}=d t^{2}-A^{2} d x^{2}-B^{2}\left(d y^{2}+d z^{2}\right),
$$

where the metric potentials $A$ and $B$ are functions of $t$ only.

The background flat metric correspondence to line element (2) is

$$
d \sigma^{2}=d t^{2}-d x^{2}-d y^{2}-d z^{2}
$$

The energy momentum tensor for the perfect fluid distribution is

$$
T_{i j}=(\rho+p) u_{i} u_{j}-p g_{i j}
$$

in which $\rho$ is the energy density and $p$ is the pressure of a perfect fluid. The four velocity time-like vector $u^{i}$ in commoving coordinate system is $u^{i}=(0,0,0,1)$ with magnitude

$$
g_{i j} u^{i} u^{j}=1
$$

The components of $T_{i j}$ are

$$
T_{11}=p A^{2}, T_{22}=T_{33}=p B^{2} \text { and } T_{00}=\rho \text { other } T_{i j}=0, i \neq j .
$$

The Rosen field equations (1) for the metric (2) and (3) are as under

$$
\begin{aligned}
& \left(\frac{\ddot{A}}{A}-\frac{\dot{A}^{2}}{A^{2}}\right)-2\left(\frac{\ddot{B}}{B}-\frac{\dot{B}^{2}}{B^{2}}\right)=16 \pi p, \\
& -\left(\frac{\ddot{A}}{A}-\frac{\dot{A}^{2}}{A^{2}}\right)=16 \pi p, \\
& \left(\frac{\ddot{A}}{A}-\frac{\dot{A}^{2}}{A^{2}}\right)+2\left(\frac{\ddot{B}}{B}-\frac{\dot{B}^{2}}{B^{2}}\right)=16 \pi \rho .
\end{aligned}
$$

where dot (.) over the head of $A$ and $B$ denotes the differentiation w. r. to $t$.

From the equations (7) and (8), we have

$$
\left(\frac{\ddot{A}}{A}-\frac{\dot{A}^{2}}{A^{2}}\right)=\left(\frac{\ddot{B}}{B}-\frac{\dot{B}^{2}}{B^{2}}\right),
$$

which on integrating yield

$$
A=k_{1} B
$$


where $k_{1}$ is positive constant.

Using the above relation (11), the equations (8) and (9) take the form

$$
\begin{aligned}
& -\left(\frac{\ddot{B}}{B}-\frac{\dot{B}^{2}}{B^{2}}\right)=16 \pi p, \\
& 3\left(\frac{\ddot{B}}{B}-\frac{\dot{B}^{2}}{B^{2}}\right)=16 \pi \rho .
\end{aligned}
$$

\title{
III. Solutions Of The Field Equations With Their Geometrical And Physical Significance
}

\author{
3.1 Model with perfect fluid
}

With the equation of state $p=\omega \rho, 0 \leq \omega \leq 1$ of perfect fluid, the equations (12) and (13) have the solution

$$
\begin{aligned}
& A=k_{1} e^{c_{1} t}, \\
& B=e^{c_{1} t},
\end{aligned}
$$

where $c_{1}$ is the constant of integration.

The volume of the model is

$$
V=A B^{2}=k_{1} e^{3 c_{1} t}
$$

Thus the required metric is

$$
d s^{2}=d t^{2}-k_{1} e^{2 c_{1} t} d x^{2}-e^{2 c_{1} t}\left(d y^{2}+d z^{2}\right)
$$

This is the LRS bianchi type I perfect fluid model in bimetric theory of gravitation. This model has volumetric exponential expansion. The model starts with non-zero volume and non-zero scale factors and the volume and scale factors increasing as time $t$ increasing and reaches infinite values at final stage.

The Hubble parameter $H$ and its directional's $H_{1}, H_{2}$ and $H_{3}$ are

$$
H=H_{1}=H_{2}=H_{3}=c_{1}
$$

The energy density $\rho$, pressure $p$, the scalar expansion $\theta$, the shear $\sigma$ and the decelerating parameter $q$ are given by

$$
\begin{aligned}
& \rho=p=0 \\
& \theta=3 c_{1}, \\
& \sigma^{2}=0 \\
& q=-1
\end{aligned}
$$

It is seen that the energy density $\rho$ and pressure $p$ in this perfect fluid model attain zero values forever indicating that the model is dust universe. The Hubble parameter $H$ and its directional's $H_{1}, H_{2}$ and $H_{3}$ are independent of time $t$ and admit constant (non-zero) values which shows that the rate of expansion is constant. Also the scalar expansion $\theta$ is independent of time $t$ and attains non-zero constant value which supports the expansion in the model. The model has zero shear. The deceleration parameter $q=-1$ shows model has accelerating phase.

Thus the LRS Bianchi type I perfect fluid model is always supports the dust universe with accelerating expansion and it is isotropize and shearless.

\subsection{Model with quintessence dark energy}

The equation of state for the quintessence model is given by

$$
p_{q}=\omega_{q} \rho_{q}, \text { where } \omega_{q} \in[-1,0]
$$

and in view of this, the equations (12) and (13) can be taken together as 


$$
\left(3 \omega_{q}+1\right)\left(\frac{\ddot{B}}{B}-\frac{\dot{B}^{2}}{B^{2}}\right)=0
$$

We bifurcate the equation of state parameter $\omega_{q}$ as

$$
\begin{array}{ll}
\left(\frac{\ddot{B}}{B}-\frac{\dot{B}^{2}}{B^{2}}\right)=0 & , \text { for } \omega_{q} \in[-1,0], \omega_{q} \neq-1 / 3 \\
\left(\frac{\ddot{B}}{B}-\frac{\dot{B}^{2}}{B^{2}}\right) \neq 0 & \text {, for } \omega_{q}=-1 / 3
\end{array}
$$

For $\omega_{q} \in[-1,0], \omega_{q} \neq-1 / 3$, the equation (25) yield

$$
B=e^{c_{1} t}
$$

and equation (11) gives

$$
A=k_{1} e^{c_{1} t}
$$

Thus the scale factors $A, B$ and the volume $V$ are given by

$$
\begin{aligned}
& A=k_{1} e^{c_{1} t}, \\
& B=e^{c_{1} t}, \\
& V=A B^{2}=k_{1} e^{3 c_{1} t},
\end{aligned}
$$

where $k_{1}$ is positive constant and $c_{1}$ is constant of integration.

The LRS Bianchi type I quintessence dark energy model (for $\omega_{q} \in[-1,0], \omega_{q} \neq-1 / 3$ ) in Bimetric theory of gravitation has volumetric exponential expansion. The volume $V$ and the scale factors $A, B$ behave in the similar way as that of the perfect fluid model (17).

The Hubble parameter $H$ and its directional's $H_{1}, H_{2}$ and $H_{3}$, energy density $\rho_{q}$, pressure $p_{q}$, the scalar expansion $\theta$, the shear $\sigma$ and the decelerating parameter $q$ obeys the values

$$
\begin{aligned}
& H=H_{1}=H_{2}=H_{3}=c_{1}, \\
& \rho_{q}=p_{q}=0, \\
& \theta=3 c_{1}, \\
& \sigma^{2}=0, \\
& q=-1 .
\end{aligned}
$$

It is seen that all these physical parameters, the Hubble parameter $H$ and its directional's $H_{1}, H_{2}, H_{3}$, the energy density $\rho_{q}$, pressure $p_{q}$, the scalar expansion $\theta$, shear $\sigma$ and the decelerating parameter $q$ having exactly similar nature as that of the physical parameters of perfect fluid model (17) whose physics explained earlier in perfect fluid model and there is no any new physics they are gaining in the model. For $\omega_{q}=-1 / 3$, the equation (26) gives

and the model is

$$
\begin{aligned}
& A=k_{1} e^{c_{1} t^{2}}, \\
& B=e^{c_{1} t^{2}}, \\
& V=A B^{2}=k_{1} e^{3 c_{1} t^{2}}
\end{aligned}
$$

$$
d s^{2}=d t^{2}-k_{1} e^{2 c_{1} t^{2}} d x^{2}-e^{2 c_{1} t^{2}}\left(d y^{2}+d z^{2}\right),
$$

which has volumetric exponential expansion starting with non-zero volume and non-zero scale factors and the volume and scale factors are infinite at later stage of time $t$.

The physical parameters are 


$$
\begin{aligned}
& H=H_{1}=H_{2}=H_{3}=2 c_{1} t, \\
& p_{q}=(-1 / 3) \rho_{q}=c_{1} / 8 \pi k, \\
& \theta=6 c_{1} t \\
& \sigma^{2}=0, \\
& q=-\left(1+2 c_{1} t^{2}\right) / 2 c_{1} t^{2} .
\end{aligned}
$$

This dark energy quintessence model for $\omega_{q}=-1 / 3$ represents dark energy star, it's energy density $\rho_{q}$, pressure $p_{q}$ are constant forever shows that there is uniform distribution of dark matter. The Hubble parameter $H$ and its directional's $H_{1}, H_{2}$ and $H_{3}$ are increasing function of time $t$ shows that the rate of expansion is increases with time $t$. Also the scalar expansion is increasing function of time $t$ which supports the model is continuously expanding. The magnitude of shear $\sigma$ is zero shows that the model is shear less. The deceleration parameter $q$ is found to be negative shows that the model has accelerating phase of expansion.

Thus the quintessence dark energy model for $\omega_{q}=-1 / 3$ is represents dark energy star starting with non-zero volume and non-zero scale factors and has volumetric exponential expansion with uniform distribution of dark matter. The model is shearless and continuously expanding with accelerating phase.

\subsection{Model with Chaplygin gas dark energy}

The equation of state for the standard Chaplygin gas is given by

$$
p_{q}=-\frac{\mu}{\rho_{q}},
$$

where $\mu$ is constant.

From equation (12) and (13), and using above equation (44), we have

$$
\begin{aligned}
& A=k_{1} e^{\frac{\sqrt{\mu \alpha}}{2} t^{2},}, \\
& B=e^{\frac{\sqrt{\mu \alpha}}{2} t^{2}},
\end{aligned}
$$

where $\alpha=\frac{(16 \pi k)^{2}}{3}$ is constant.

$$
\begin{gathered}
V=k_{1} e^{\frac{3 \sqrt{\mu \alpha} t^{2}}{2}}, \\
d s^{2}=d t^{2}-k_{1} e^{\sqrt{\mu \alpha} t^{2}} d x^{2}-e^{\sqrt{\mu \alpha} t^{2}}\left(d y^{2}+d z^{2}\right)
\end{gathered}
$$

This is LRS Bianchi type I Chaplygin gas dark energy model in bimetric theory of gravitation and having the same geometry, same volume and same scale factors as that of the quintessence model (38) corresponding to $\omega_{q}=-1 / 3$.

The physical parameters, like the Hubble parameter $H$ and its directional's $H_{1}, H_{2}$ and $H_{3}$, the energy density $\rho_{c}$, pressure $p_{c}$, the scalar expansion $\theta$, the shear $\sigma$ and the decelerating parameter $q$ are as follows

$$
\begin{aligned}
& H=H_{1}=H_{2}=H_{3}=\sqrt{\mu \alpha} t, \\
& p_{c}=(-1 / 3) \rho_{c}=\sqrt{\mu \alpha} / 16 \pi k, \\
& \theta=3 \sqrt{\mu \alpha} t, \\
& \sigma^{2}=0, \\
& q=-\left(1+t^{2} \sqrt{\mu \alpha}\right) / t^{2} .
\end{aligned}
$$


It is to be noted that the results of all the physical parameters, volume $V$, the Hubble parameter $H$ and its directional's $H_{1}, H_{2}$ and $H_{3}$, energy density $\rho_{c}$, pressure $p_{c}$, scalar expansion $\theta$, shear $\sigma$ and the decelerating parameter $q$ matched with those of quintessence model (38) corresponding to $\omega_{q}=-1 / 3$ and therefore they admit the same physics as remarked in quintessence model (38) for $\omega_{q}=-1 / 3$ and there is no any new ideas they contributing in physical properties. Therefore it is observed that this Chaplygin gas model (48) is goes over to quintessence model (38) for $\omega_{q}=-1 / 3$ along with its geometrical and physical properties. It is remarkable point that the Chaplygin gas model (48) and the quintessence model (38) corresponding to $\omega_{q}=-1 / 3$ both represents dark energy star and filled with uniform distribution of dark matter, they continuously expanding with accelerating expansion and are shearless. and shearless.

All these perfect fluid model, quintessence model and the Chaplygin gas model are isotropize in nature

\section{Summary}

1) LRS bianchi type I models have been investigated, in related to perfect fluid, quintessence dark energy and standard Chaplygin gas by solving Rosen's field equations in Bimetric theory of gravitation.

2) LRS bianchi type I perfect fluid model has volumetric exponential expansion starting with non-zero volume and non-zero scale factors.

3) LRS bianchi type I perfect fluid model is always supports the dust universe with accelerating expansion and it is isotropize and shearless.

4) LRS bianchi type I quintessence dark energy model, for $\omega_{q} \neq-1 / 3$ is goes over to perfect fluid model and its geometry and all the physical parameters behave in similar fashion as that of perfect fluid model. This quintessence dark energy model is also represents dust universe with accelerating expansion and it is isotropize and shearless.

5) LRS bianchi type I quintessence dark energy model (38) corresponding to $\omega_{q}=-1 / 3$ also has volumetric exponential expansion starting with non-zero volume and non-zero scale factors and it represents dark energy star.

6) This dark energy quintessence model corresponding to $\omega_{q}=-1 / 3$ filled with uniform distribution of dark matter and it is continuously expanding with accelerating phase.

7) LRS bianchi type I standard Chaplygin gas model (48) has the same geometry, same volume and same scale factors as that of quintessence model (38) corresponding to $\omega_{q}=-1 / 3$ and all its physical parameters admits the same physics as remarked in the literature of quintessence model for $\omega_{q}=-1 / 3$ and there is no any new idea they contributing in physical properties.

8) It is remarkable point that the perfect fluid model and quintessence model for $\omega_{q} \neq-1 / 3$ admits the same physics.

9) It is realized that the quintessence dark energy model for $\omega_{q}=-1 / 3$ and standard Chaplygin gas model, both represents dark energy star.

10) All these perfect fluid model, quintessence model and standard Chaplygin gas model are isotropize in nature and shearless.

\section{Conclusion}

LRS bianchi type I models corresponding to perfect fluid, quintessence and standard Chaplygin have been investigated by solving the Rosen's field equations in bimetric theory of gravitation. It is observed that the geometry of perfect fluid model is coincide with the geometry of quintessence dark energy model for $\omega_{q} \in[-1,0], \omega_{q} \neq-1 / 3$. It is also realized that the geometry of quintessence dark energy model for $\omega_{q}=-1 / 3$ is similar to geometry of standard Chaplygin gas model. Other geometrical and physical properties of the models are studied. 
It is pointed out that all these perfect fluid, quintessence and standard Chaplygin gas dark energy models are expanding with accelerating expansion and our opinion is agreed with, the observational data [5-11], as they remarked that the universe is continuously expanding with accelerating phase.

\section{Acknowledgments}

The authors express their sincere thanks to UGC, New Delhi, for providing financial assistance to carry out this work under Major Research Project.

[1]. A.G. Riess, et al., Astrophysical Journal, 607, 2004, 665.

[2]. D.J. Eisensteinet al., Astrophysical Journal, 633, 2005, 560.

[3]. P. Astieret al., Astronomy and Astrophysics, 447, 2006, 31.

[4]. D.N. Spergel et al., Astrophysical Journal Supplement Series, 170, 2007, 3771.

[5]. A.G. Riess, A.V. Filippenko, P. Challis,A. Clocchiatti, A. Diercks, P.M.Garnavich, R.L. Gilliland, C.J. Hogan, S.Jha, R.P.Kirshner, B.Leibundgut, M.M. Phillips, D. Reiss, B.P. Schmidt, R.A. Schommer, R.C. Smith, J.Spyromilio, C. Stubbs, N.B.Suntzeff andJ.Tonry 3: Observational evidence from supernovae for an accelerating universe and a cosmological constant. Astronomical Journal, 116, 1998, 1009

[6]. S. Perlmutter, G. Aldering, G.Goldhaber, R.A. Knop, P. Nugent, P.G. Castro, S.Deustua, S.Fabbro, A.Goobar, D.E. Groom, I.M. Hook, A.G. Kim, M.Y. Kim, J.C. Lee, N.J.Nunes, R. Pain, C.R. Pennypacker, R.Quimby, C.Lidman, R.S. Ellis, M. Irwin, R.G. McMahon, P. Ruiz-Lapuente, N. Walton, B. Schaefer, B.J. Boyle, A.V.Filippenko, T. Matheson, A.S.Fruchter, N.Panagia andH.J.M. Newberg 2 et al.: Measurements of $\Omega$ and $\Lambda$ from 42 high-redshift supernovae, Astrophysical Journal, 517, 1999, 565.

[7]. N.A. Bahcall, J.P.Ostriker, S. Perlmutter andP.J. Steinhardt: The cosmic triangle: revealing the state of the universe, Science, 284, 1999, 1481-1488.

[8]. M. Tegmark, D. Eisenstein, M. Strauss, D. Weinberg, M. Blanton, J.Frieman, M.Fukugita, J. Gunn, A. Hamilton, G. Knapp, R. Nichol, J.Ostriker, N.Padmanabhan, W. Percival, D. Schlegel, D. Schneider, R.Scoccimarro, U.Seljak, H.Seo, M. Swanson, A.Szalay, M.Vogeley, J.Yoo, I.Zehavi, K.Abazajian, S. Anderson, J.Annis, N.Bahcall, B. Bassett, A. Berlindand J. Brinkmann12, et al.: Cosmological constraints from the SDSS luminous red galaxies. Physics Review D, 74, 2006, 1-34.

[9]. C.L. Bennet, M. Halpern, G.Hinshaw, N.Jarosik, A.Kogut, M. Limon, S.S. Meyer, L. Page, D.N.Spergel, G.S. Tucker, E.Wollack, E.L. Wright, C. Barnes, M.R. Greason, R.S. Hill, E. Komatsu, M.R.Nolta, N.Odegard, H.V. Peirs, L. Verde andJ.L. Weiland: FirstyearWilkinson Microwave Anisotropy Probe Observations: preliminary maps and basic results, Astrophysical Journal Supplement Series, 148, 2003, 1-21.

[10]. G. Hinshaw, J.L.Weiland, R.S. Hill, N.Odegard, D. Larson, C.L. Bennett, J. Dunkley, B. Gold, M.R.Greason, N.Jarosik, E.Komatsu, M.R. Nolta, L. Page, D.N.Spergel, E.Wollack, M. Halpern, A.Kogut, M. Limon, S.S. Meyer, G.S. Tucker andE.L. Wright: Five-yearWilkinson Microwave Anisotropy ProbeObservations: data processing, sky maps and basic Results, Astrophysical Journal Supplement Series, 180, 2009, 225-245.

[11]. M.R. Nolta, J. Dunkley, R.S. Hill, G.Hinshaw, E. Komatsu, D. Larson, L. Page, D.N.Spergel, C.L. Bennett, B. Gold, N.Jarosik, N.Odegard, J.L.Weiland, E.Wollack, M. Halpern, A.Kogut, M. Limon, S.S. Meyer, G.S. Tucker, E.L. Wright 2: Five-year Wilkinson Microwave Anisotropy Probe Observations: angular power spectra, Astrophysical Journal Supplement Series, 180, 2009, 296-305.

[12]. B. Ratra andJ. Peebles, Physics Review D, 37, 1988, 321.

[13]. C. Wetterich, Nuclear Physics B, 302, 1988, 668.

[14]. R.R. Caldwell, Physics Letter B, 545, 2002, 23.

[15]. Y.H. Wei andY. Tian, Classical Quantum Gravity, 21, 2004, 5347.

[16]. E. Elizalde, S. Nojiri and S.D.Odinstov, Physics Review D, 70, 2004, 043539.

[17]. S. Nojiri, S.D. Odinstov andS.Tsujikawa, Physics Review D, 71, 2005, 063004.

[18]. T. Padmanabhan, Physics Review D, 66, 2002, 021301.

[19]. A. Sen, Journal of High Energy Physics, 04, 2002, 048.

[20]. A. Kamenshchik, U. Moschella andV.Pasquier, Physics Letter B, 511, 2001, 265.

[21]. S.D. Katore, D.V. Kapse andG.B.Tayade, International Journal of Theoretical Physics, 50,2011, 3299.

[22]. E.J. Copeland, M. Sami andS. Tsujikawa, International Journal of Modern Physics D, 15, $2006,1753$.

[23]. B. Saha, Chinese Journalof Physics, 43, 2005, 1035.

[24]. T. Singh andR. Chaubey, Astrophysics Space Science, 319, 2009, 149.

[25]. A. Dev, J.S. Alcaniz and D. Jain, Physics Review D, 67, 2003, 023515.

[26]. M.R. Setare, International Journal of Modern Physics D, 18, 2009, 419.

[27]. M.C. Bento andO. Bertolami and A. Sen, Physics ReviewD, 66, 2002, 043507.

[28]. Z.K. Guo andY.Z. Zhang, Physics Letter B, 645, 2007, 326

[29]. Abdusattar andS.R. Prajapati, Astrophysics Space Science, 332, 2011, 455.

[30]. M. Shen and L. Zhao, Astrophysics Space Science, 337, 2012, 753.

[31]. N. Rosen, Plenum Press, London, 455, 1974.

[32]. N. Rosen, Plenum Press, London, 273, 1977.

[33]. T.M. Karade, Indian Journal ofPure and Applied Mathematics, 11(9),1980, 1202.

[34]. M. Isrelit, General Relativity and Gravitation, 13(7),1981, 681.

[35]. D.R.K. Reddy and N.VenkateswaraRao, Astrophysics Space Science, 257,1998, 293.

[36]. R. Bali and Anjali, Astrophysics Space Science, 302,2006, 201.

[37]. R. Bali andS. Dave, Astrophysics Space Science, 288,2003, 503.

[38]. R. Bali and R.D.Upadhaya, Astrophysics Space Science, 283,2003, 97.

[39]. R. Bali andD. Singh, Astrophysics Space Science, 300,2005, 387.

[40]. R. Bali and U.K.Pareek, Astrophysics Space Science, 312,2007, 305.

[41]. S.D. Katore andR.S.Rane,Pramana Journal of Physics, 67(2),2006, 237

[42]. G.S. Khadekar and S.D.Tade,Astrophysics Space Science, 1998, DOI 10.1007\ S10509-00794102.T

[43]. M.S. Borkarand S.S.Charjan, International Journal of Applied Mathematics, 22(3), 2009, 445.

[44]. M.S. Borkar and S.S. Charjan, Journal of Indian Academy of Mathematics, 32(1), 2010. 
[45]. M.S. Borkar and S.S. Charjan, An International Journal of Application and Applied Mathematics, 5(1),2010, 96109.

[46]. M.S. Borkar and S.S. Charjan, An International Journal of Application and Applied Mathematics, 5(2), $2010,1660$.

[47]. M.S. Borkar and S.S. Charjan andV.V. Lepse, International Journal of Applied Mathematics, 28(2),2013, 2051.

[48]. N.P. Gaikwad, M.S.Borkar and S.S.Charjan,Chinese Physics Letter, 28(8),2011, 089803. 